\title{
Applications of Geiger-mode APDs in Astroparticle Physics Research
}

\section{E. Lorenz ${ }^{1}$}

Max Planck Institute for Physics and Institute for High Energy Physics, ETH Zurich

Foehringer Ring 6, D80805 Munich, Germany and ETH Zurich, Hoengger Berg, Zurich, Switzerland

E-mail: e. Iorenz@mac.com

Abstract. : The novel Geiger mode avalanche photodiodes (G-apd) offer an alternative to photomultipliers (PMT) in many Astroparticle Physics experiments and promise a significant improvement in sensitivity and in many cases a lower energy threshold. Three examples will be discussed. 


\section{Introduction}

The earth is constantly bombarded by energetic particles from outer space. These particles, historically called Cosmic Rays (CR), are 'messengers' of high energy processes of the relativistic, i.e., non-thermal universe. Since a few decades the study of the relativistic universe becomes more and more important as its understanding is needed to get a complete picture of our cosmos and its evolution. The study of cosmic high energy particle processes is named Astroparticle physics (APP), which is a new area of fundamental research carried out by ground-based, balloon or satellite borne detectors.

The currently most active areas of Astroparticle Physics are:

- High energy (HE) and very high energy (VHE) Gamma-Ray $(\gamma)$ astronomy

- Study of the highest energy $\left(>10^{19} \mathrm{eV}\right)$ cosmic particles

- Neutrino astronomy

- Dark matter searches (WIMPs, Neutralinos.....)

- Nuclear Astrophysics

- (Gravitational wave physics)

Besides the mostly astronomical observations one searches also for new particles not observed by terrestrial High Energy Physics (HEP) experiments such as for example for WIMPS, Neutralinos, Topological Defects, Relic Particles or the Graviton. Most known CRs are charged particles. They are not well suited as messenger particles, except above around $10^{19}$ $\mathrm{eV}$ because they are mostly deflected by galactic and earth magnetic fields and thus cannot be traced back to their origin, i.e., to stellar environments where they are assumed to be generated. Cosmic $\gamma$ s, although only a minuscule fraction of the CRs and, as well as, vs are unaffected by cosmic magnetic fields and can be extrapolated to their origin. Thus they are important messengers of cosmic particle processes.

Observation of cosmic particles is in many aspects a challenge for experimenters:

- APP is an observational science. One cannot control the initial parameters such as time of occurrence of important processes or the initial energy or chemistry of the messengers.

- The fluxes of energetic cosmic messengers are generally low and the flux drops normally strongly with increasing energy. In the currently most extreme low but still observable flux one detects with appropriate detectors extreme high energy particles $\left(>10^{19} \mathrm{eV}\right)$ with a flux of one particle $/ \mathrm{km}^{2}$ and 100 years. As consequence of the low fluxes and high energies one needs huge detector volumes acting as calorimeters.

Another consequence of the required large detector volumes is that one has to rely mostly on 'natural' materials such as the atmosphere, water or ice, in which the calorimetric absorption generates observable, long flying photons, either by scintillation (only in the atmosphere) or Cherenkov light. Some future experiments of restricted volumes, i.e. detector masses $(<100000$ tons) consider liquid organic scintillators or liquid noble gasses such as Argon. In summary nearly all detectors for high energy cosmic particles rely on efficient photon detection. Obviously, these photon detectors must have a large active area, high effective quantum efficiency (QE), be able to detect single photons and have a very good time resolution in the 
nsec domain. In many cases fine pixelized photon detectors are needed. In addition they should be rather robust against adverse environmental conditions and affordable in price. Up to now only photomultipliers (PMT) could fulfil most of the requirements, although the low peak QE of up to $25-30 \%$ around $400 \mathrm{~nm}$ is one of the most limiting parameters. Low cost photon detectors of high photon detection efficiency (PDE) would have a major impact on the field allowing to improve the sensitivity and/or lower the detection threshold of most experiments. Up to now solid state photodetectors, in spite of high QE, were unsuited because of the inability to detect single photons, relatively low speed, high noise and small area. The currently developed solid state photon detectors, based on p-n silicon avalanche structures, have the promise to improve many experiments. In the next chapter some key parameters of these Geiger-mode avalanche photodetectors (G-apd) will be briefly reviewed and their features compared with PMTS. In the following chapters the possible use in so-called imaging air Cherenkov telescopes for groundbased very high energy $\gamma$-astronomy, in satellite borne air fluorescence detectors for the study of the highest energy CRs and in hybrid photon detectors for $v$ - detectors will be discussed.

\section{G-apds as a possible replacement for PMTs in APP experiments}

G-apds, solid-state light detectors with high internal gain and single photon response were originally developed in the eighties in the former Soviet Union. After the breakdown of the Soviet Union research continued in Russia by several groups, see for example [1], [2] and [3], with the aim to produce an inexpensive high efficient photon detector. Recently, the development of G-apds (also called SiPM, MPPC, MPGM-APD, SSPM, MRS-APS, MKPD..) is accelerating because of significant performance improvements, of a large potential for many possible applications and the potential to modify easily the structure and to adopt them to quite different needs (see the many different contributions to this conference). Fig 1 shows an isometric view of the basic structure of a G-apd. A G-apd is composed of an array of small avalanche photodiodes (cells) combined to form a macroscopic unit (typically 100 to 4000 small separated apds per $\mathrm{mm}^{2}$ ). Each cell, connected to a common grid bus by an integrated polysilicon resistor (see insert fig 1), operates in limited Geiger mode, i.e. a few Volts above breakdown voltage. In this mode of operation an (photo)electron initiates a local avalanche breakdown confined to a cell, i.e., the electron "triggers" the cell. The resistor limits the external charging current for the junction and eventually quenches the avalanche once the cell capacity has been discharged.

For each Geiger breakdown the output signal of a cell has nearly the same shape and charge. Therefore the output signal on the bus is the superposition of the standardized signals of all fired cells. In case of not too intense light flashes the number of fired cells is in first order proportional to the number of photons thus compensating for the missing dynamic range of a single cell Geiger mode APD. Further details can be found in [4]. The main advantages of Gapds compared to PMTs are:

- A high PDE exceeding for some designs that of PMTs by a factor up to 3-4

- Ability to detect single photons and generating a fixed signal for a single PE/cell

- Very compact design, insensitive to magnetic fields

- A low operation voltage wrt. PMTs, typically below $100 \mathrm{~V}$ for high gain $\left(10^{4}-10^{6}\right)$ 
- Very robust, are not damaged when accidentally exposed under bias to ambient light

- Long-term prospects for easy fabrication and low production costs

Disadvantages are:

- Currently a small size, requiring summing amplifiers for larger sensor area

- High dark noise rate (100 kHz-many $\mathrm{MHz} / \mathrm{mm}^{2}$ at room temperature)

- Optical cross-talk (can be partially suppressed by grooves)

- Nonlinearity when the number of photons exceeds the number of cells

- A rather long time for recharging the fired cells (nonfired cells unaffected)

Initially, nearly all G-apds were based on an n-on-p structure with high sensitivity for red light. Recently, high sensitivity, low noise p-on-n structures have been produced with the peak PDE around 400-500 nm, e.g. being well suited for the detection of Cherenkov light.
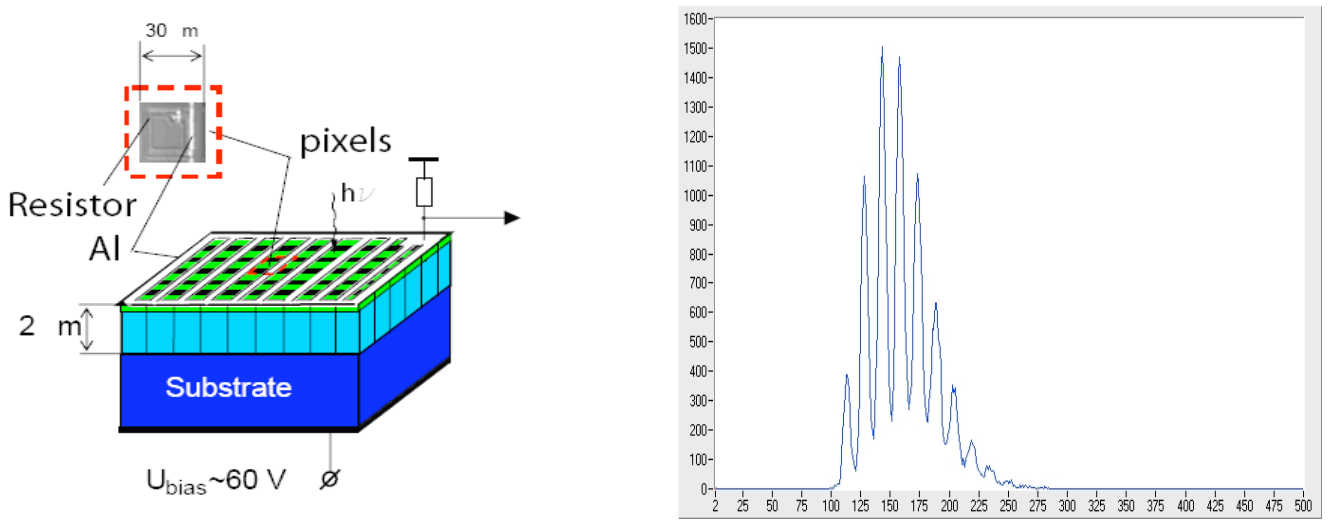

Figure 1 A) Isometric view of a simplified G-apd. The small insert shows a picture of one of the Geiger diodes of which the G-apd is composed. The Geiger diode is connected via a small polysilicon resistor to the grid, which serves as common readout for all cells (courtesy B. Dolgoschein). B) Example of the pulse height distribution when illuminating a G-apd by a low intensity light flash.

\section{Possible use in ground-based VHE $\gamma$-ray Astronomy}

Ground-based very high energy (VHE) $\gamma$-astronomy has entered a very productive discovery phase. The 'window' was opened 1989 by the discovery of TeV $\gamma$ s from the CRAB nebula [5]. Since then more than 40 sources have been discovered and many fundamental results retrieved. Nearly all studies have been carried out by so-called imaging atmospheric Cherenkov telescopes (IACT), which allow one to detect the very faint Cherenkov light flashes generated by particle showers in the atmosphere. These showers are initiated by energetic cosmic particles, mostly by charged hadrons but also rarely by $\gamma$-rays hitting the upper atmosphere. The IACT technique allows an efficient $\gamma /$ hadron separation and an efficient directional and energy measurement with a typical precision of $0.1^{\circ}$ and $20 \%$ resolution, respectively. IACTs view the night sky around potential sources by means of a large mirror collecting the shower Cherenkov light and focussing it onto an array of photon detectors in the focal plane. By means of the analysis of the Cherenkov light pattern, reflecting differences in hadronic and $\gamma$-showers, one can filter out the rare $\gamma$ - signals. Because of the ultra-fast C-light flashes a detection against the large night sky light background ( $\mathrm{NSB}, \mathrm{O}$ few $10^{12} / \mathrm{m}^{2} \mathrm{sec}$ sterad), even against modest moon shine, is possible. A key quantity for high sensitivity and a low detection threshold is the product of the mirror area times the PDE of the fine pixelized photon 
detector. While for optical, technical and cost reasons an increase in mirror area beyond the currently largest ones is difficult to realize an increase in PDE by means of the use of G-apds is very promising. First tests of detecting Cherenkov light have been reported confirming a significant improvement in photon detection when using prototype G-apds [6], [7]. Fig 2 shows a Cherenkov light flash seen by four $3 \times 3 \mathrm{~mm}^{2}$ Hamamatsu MPPCs and 3 nearby PMT pixels ( 7 $\mathrm{cm}^{2}$ area each) of the MAGIC camera, [7]. Fig 3. shows a comparison of the folded spectrum of the Cherenkov spectrum times the PDE of various photosensor material times the typical optical parameters of the optics of an IACT (mirror reflectivity, window transmission, stray light losses, etc.). Using recently published data of Hamamatsu G-apds with 100 cells $/ \mathrm{mm}^{2}$ the integrated gain in photon detection efficiency is expected to be three times that compared to classical bialkali PMTs.
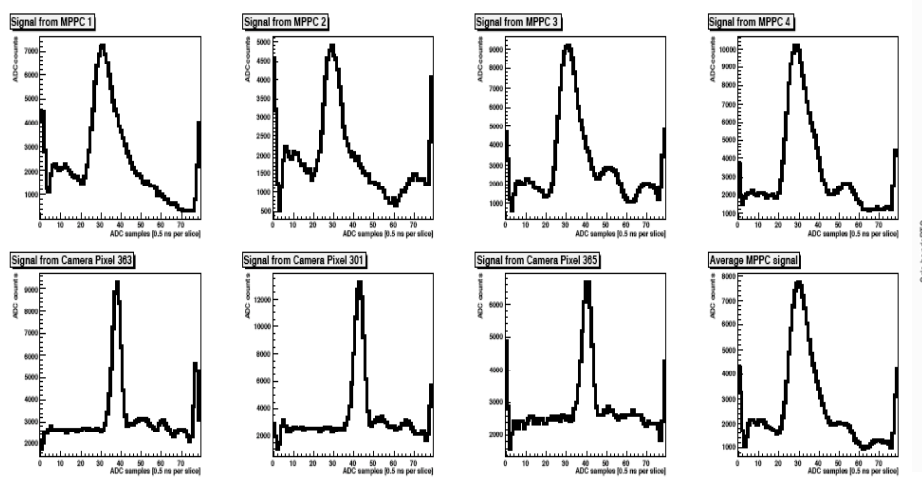

Fig 2. A Cherenkov light flash recorded by a cluster of $4 \mathrm{G}$-apds $(3 \times 3 \mathrm{~mm}$ Hamamatsu MPPC prototypes) and 3 PMTs (lower left row) and the sum signal of the G-apds (lower right panel) installed on the MAGIC telescope. [8]. Signals recorded by a $2 \mathrm{GHz}$ F-ADC.

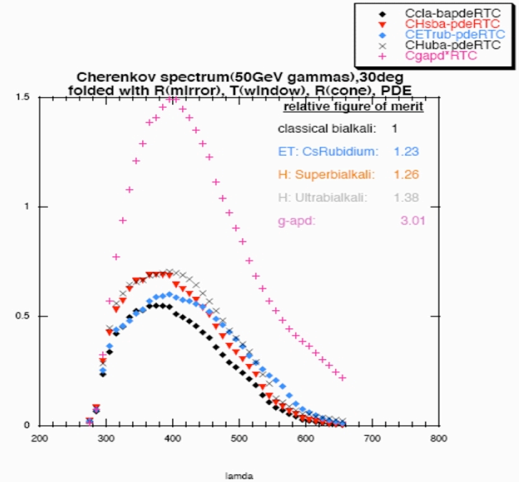

Fig 3. $50 \mathrm{GeV}$ em Cherenkov spectrum folded by typical opt. parameters of an IACT multiplied by the PDE of some photon detectors

\section{Possible use of G-apds in extreme high energy Cosmic Ray Studies}

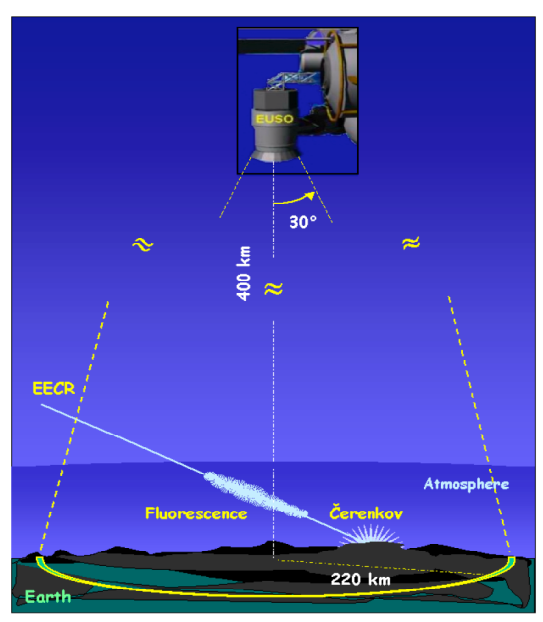

Fig 4: Artist view of the EUSO detector.

Another very active area in APP is the study of extreme high energy $\left(>10^{19} \mathrm{eV}\right)$ CRs. These particles are extremely rare (O 1 particle $/ 100 \mathrm{~km}^{2}$ and year). Current ground-based detectors are at most $2000 \mathrm{~km}^{2}$ large (AUGER, HIGH RES FLY's EYE) detecting either the shower tail particles or the weak fluorescent light generated by charged shower particles, which excite $\mathrm{N}_{2}$ molecules of the atmosphere. The latter method can be carried out by satellite borne large angle telescope. Currently, a few projects are under study: EUSO, JEM-EUSO, S-EUSO, AIR-WATCH, OWL. These telescopes, either coupled to the Space Station or as free-flyers, can detect signals from up to $10^{6} \mathrm{~km}^{2}$ area with $\approx 10-20 \%$ duty cycle. The basic detector comprises a large imaging optics, such as a

Fresnel lens or a mirror, that images the fluorescent light pattern of the shower onto a large plane of fine pixelized photosensors ( $\mathrm{O}$ few $10^{5}-10^{6}$ pixels) and the associated trigger and 
readout electronics. Fig 4 shows an artist view of the EUSO detector coupled to the Space Station. The original design was based on a large matrix of multipixel PMTs of typically 12$15 \%$ peak PDE. Replacing the PMTs by G-apds promises a large increase in sensitivity $(>4)$ as well as lower weight, lower power consumption, no need of HT and low risk of damage when exposed accidentally to ambient light when biased. Obviously, some problems have to be solved, such as a reduction of noise and optical cross-talk. Also, G-apds are not yet spacequalified and their radiation hardness to survive solar flares and passage of the Van Allen belt must be proven. The most ambitious project, S-EUSO, uses G-apds as baseline design [9].

\section{G-apds as secondary readout for smart PMTs for Neutrino Detectors.}

The third most active area in APP is the study of cosmic v's. This search, extending from the $\mathrm{MeV}$ energy range (i.e., mostly solar $v$ 's) to the highest energies $\left(>10^{20} \mathrm{eV}\right)$ covers both astronomical and fundamental $v$ parameter studies. Due to the very small $v$ interaction crosssection ultralarge detectors of high density are needed (water, ice, large volumes of scintillating materials). Again, only light produced by scintillation or by Cherenkov radiation allows a costeffective detection of $v$-interaction products in large volumes, but it is obvious that a large number of large area (O 15-50 cm Ø) photosensors of high QE, excellent single electron response (SER) and low intrinsic noise is needed. G-apds are not yet able to fulfil the large area and low noise requirements. Only large diameter PMTs are suited but they have as serious drawback a rather poor photoelectron (PE) collection efficiency when focussing PEs onto the dynode system. The alternative to increase the first dynode area results in very poor timing. Some time ago an alternative PMT was proposed and also a small series built, the so-called Smart PMT $[10,11,12]$, which is basically a light amplifier. The dynode complex is replaced by a small scintillator overcoated by a very thin aluminium layer acting both as a high voltage

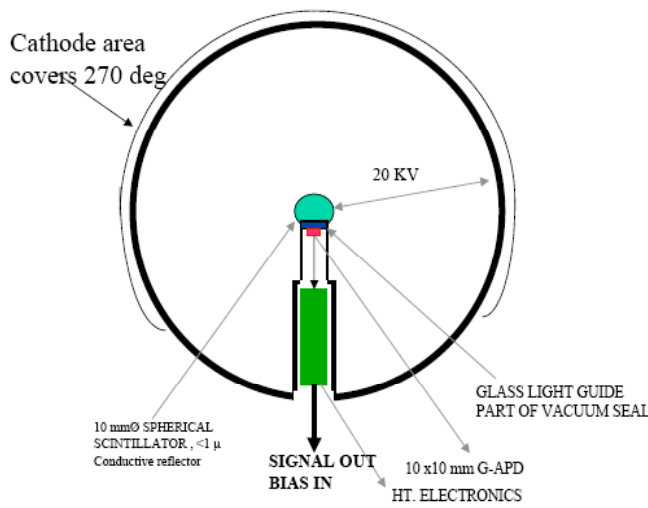

Fig.5. A design concept of a Smart PMT anode and light shield. Photoelectrons are accelerated by $20-30 \mathrm{KV}$ and generate a light signal in the scintillator, which is viewed from the backside by another light sensor (a PMT in the original design). Fig 5 shows a proposal where the secondary PMT is replaced by a large area (or a matrix) G-apd. Such a device should have basically $100 \%$ PE collection efficiency (except backscatter losses) and, due to a perfect spherical geometry, a very low timing jitter. Also, no magnetic shielding is required because of the high photoelectron acc. voltage (The cathode is on ground). Replacing the secondary PMT by a G-apd simplifies considerably the detector and should result in a major cost reduction. Also, it should allow a very compact construction such as a nearly perfect spherical geometry, which can be directly be immersed in shallow water (scintillator) detectors of hydrostatic pressure. The readout idea has been tested using an early Smart PMT construction, the so-called Quasar, by using a small 3x3 $\mathrm{mm}$ prototype G-apd [13] of below 
$20 \%$ PDE. Fig 6a shows an oscilloscope recording from a 2 photoelectron (PE) signal (note the fluctuations of the light emission from the $40 \mathrm{nsec}$ decay time scintillator) and fig. $6 . \mathrm{b}$ the pulse height distribution when the cathode is illuminated by a mean of $<2$ PE $>$.

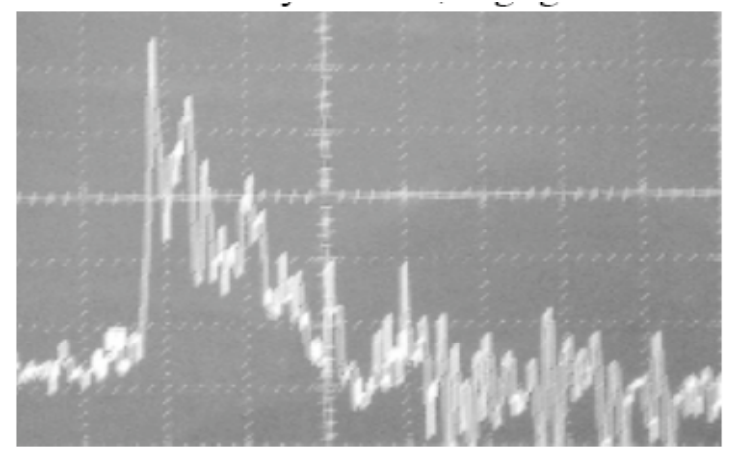

Fig. 6 a) oscilloscope screen shot for a 2 PE signal

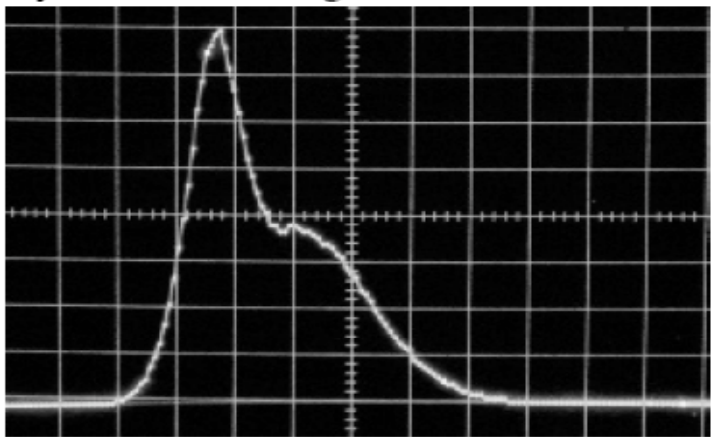

b) pulse height distribution when illuminating the PMT Cathode by alight pulse generating a $<2$ PE $>$ signal

\section{Conclusions}

G-apds have very promising features and can be used as a replacement of PMTs in many applications in APP experiments. The above shown examples of possible applications demonstrate that they can considerably improve detector performances such as increased sensitivity, lower thresholds or considerable cost savings. In summary, no other recently developed high speed detector has such a large potential for photon detection in many applications as the novel G-apds.

\section{References}

[1] B. Dolgoschein et al., NIM A504 (2003), 48.

[2] D. Bisello et al., NIM A 367 (1995) 212.

[3] V. Golovin et al., NIM A 518 (2004) 560.

[4] D. Renker, NIM A567 (2006) 48.

[5] T. C. Weekes et al., ApJ 342 (1989).

[6] A. Biland et al., contributed paper to the Vienna Inst. Conf., Feb 2007.

[7] N. Otte et al., contribution \# 1070 to the XXX ICRC 07, Merida, Mexico (2007).

[8] E. Lorenz. New A. Rev., 48, 339 (2004).

[9] S-EUSO proposal, submitted to the ESA Cosmic Visions 2025-2025.

[10] P. Bosetti et.al., Procs. XXIII ICRC (1993).

[11] R. Bagduev et al., NIM A420 (1999) 138.

[12] A. Braem et al., CERN preprint CERN -PH-EP/2006-025.

[13] E. Lorenz, D. Ferenc., NIM A 572 (2007),434. 